\title{
The therapeutic approach of the odontal pathology of the temporary teeth - theoretical and practical aspects
}

\author{
Mirel Toma ${ }^{1}$, Florentina Radu², Gabriel Ciochinda', Cristina Nicoleta Mihai', \\ Sorin Nicolae Popescu', Viorel Stefan Perieanu', Ileana Ionescu', Madalina Violeta \\ Perieanu', Magdalena Natalia Dina', Olivia Popoviciu', Raluca Costea², \\ Liliana Burlibasa ${ }^{3}$, Corina Marilena Cristache ${ }^{1}$, Mihaela Rodica Pauna ${ }^{1}$, \\ Luminita Oancea', Rodica Luca' ${ }^{1}$ \\ 1"Carol Davila” University of Medicine and Pharmacy, Bucharest, Romania \\ ${ }^{2}$ Private dental practice, Bucharest, Romania \\ ${ }^{3}$ Faculty of Biology, University of Bucharest, Romania
}

\begin{abstract}
Introduction. In the growing process of each individual, there are 4 essential stages that must be very well known by the pediatric dentist, when intervening on these changes: the stage named the age immediately after birth; pre-school age; the stage of mixed dentition and the age period after 12 years.

Purpose. Starting from the existence of these 4 stages of the growing process of each individual, in this material we tried a brief presentation of some aspects, both theoretical and practical, regarding the therapeutic approach in the dental pathology of the temporary teeth.

Material and method. The study was conducted through the collaboration of 35 dentists from all over the country based on a questionnaire consisting of 6 questions.

Result and discussion. Following the application of the questionnaire we were able to create an image of the way in which the dental pathology of the permanent teeth is therapeutically approached by the practitioners included in the study.

Conclusion. Dental lesions in the temporary dentition should be evaluated and treated with great care, to prevent the medium and long term complications of permanent dentition.
\end{abstract}

Keywords: temporary dentition, dental pathology, pulpal complications

\section{INTRODUCTION}

As it is well known, in the development of the dento-maxillary system of the people from the contemporary society, there are many tendencies, which generally favor the appearance of a deficient function characterized by the existence of abnormal relations, followed by the consecutive installation of the odontal and pathological processes.

Specifically, in the growing age of each individual, we find significant deviations from the morphology and function of the dento-maxillary system, these actually reflecting the factors that modify, affect and even condition the growth process of that individual.
Starting from these goals, the dental assistance, in our case the pediatric dental assistance, can be defined, as both a preventive assistance, but also to ensure the treatment of these morpho-functional imbalances, as well as of some dental and periodontal pathological processes of a lower or greater amplitude (1-5).

\section{PURPOSE}

In the growth flow of each individual, we notice 4 essential stages for the constituent elements of the dento-maxillary system, which must be extremely well known not only by the pediatric den- 
tist, but by any dentist, when intervening on these changes (1-5):

- The stage named and the age immediately after birth;

- The second stage up to the age of 6 years, is also called the preschool age or the age of the temporary dentition;

- The third stage, also called the period between 6 and 12 years or the stage of mixed dentition, is actually the period of changing the temporary dentition with the permanent dentition;

- The fourth stage, also called the age period after 12 years, when we talk about definitive dentition.

Starting from the title of the material, it can be observed that, the period that interests us is in fact the period of the temporary dentition, period up to the age of 12 years, period that overlaps with the preschool age and the stage of the mixed dentition. Thus, the purpose of this material is in fact the familiarization, but especially the implementation of both theoretical notions, as well as therapeutic procedures on the temporary dentition, among the dental practitioners.

\section{MATERIAL AND METHOD}

Preschool age, age up to 6 years or age of temporary dentition, is dominated by functional and nutritional factors. Thus, at the time of the temporary teeth eruption, special attention will be paid to the way in which the functions of the dento-maxillary system are performed (1-5).

The defective eating habits engraved to children at preschool age by parents and kindergarten teachers, together with hereditary transmissions regarding the oral dental tissues shortcomings, in fact determines the unfavorable functional conditions for carrying out mastication act and the harmonious development of the dento-maxillary system (1-5).

At the same time, up to the age of 6 years, the oral and dental hygiene is an extremely important factor both in terms of direct effects, and especially of the indirect effects, by fixing habits. However, this is very difficult to achieve, due to the child's scattered attention, but it is easy to set the brushing reflex at a certain time of day (1-8).

From the point of view of the dental pathology, it is observed in this interval of the preschool age, the presence of the dental caries with an important dysfunctional role in the imbalance of the dento-maxillary system. To include this stage of the preschool age in the activity of the pediatric dentist, presents a special advantage to ensure the normal activity of the dento-maxillary system, its morphology, as well as correct occlusal-articular relationship. Tooth decay treatment for the temporary dentition must ultimately lead, from a biological point of view, to healing, and from a morphological point of view, to the rendering of the functional form, which ensures the quality and correctness of the therapy (1-8).

The period of the mixed dentition (age 6-12 years) represents the stage of changing the temporary dentition with the permanent dentition and comprises 2 phases (1-8):

- Between 6 and 9 years, the stage of changes in the frontal area;

- Between 9 and 12 years, the stage of changes in the lateral areas, influenced by the eruption of the molar of 12 years.

But, what characterizes this age stage, is the carious pathology with a very high intensity index, which contributes to the installation of some malfunctions of the dento-maxillary system. The highest decay index, relative to age, is tooth decay in the lateral areas (canines - temporary molars), between 6 and 9 years (1-5).

Changes in the form and relationships produced by caries in these areas are of the utmost importance in directing the relationships between the evolving arches. Therefore, the complicated dental injuries existing both in the temporary teeth and in the permanent teeth, which require a special effort to preserve these teeth, forces to a correct morphofunctional restoration, resistant in time, important in the development of the dento-maxillary system (1-8).

At the same time, the morphological changes from the level of the temporary dentition in the lateral zones, will favor the mesial migrations of the distal teeth. In this regard, it is recommended to treat and conserve as much as possible the temporary dentition (including root remains, when the situation permits), in order to stop the mesial migrations. It is necessary to insist on the conservative treatment of carious lesions with localization to the temporary teeth, from the support areas (1$8)$.

Starting from these important notions in order to perform dental therapeutic acts for the temporary dentition of patients up to 12 years old, we made up a questionnaire composed of 6 questions, which we applied to a number of 35 dentists, who work in the private environment from various big cities in Romania, such as the cities of Bucharest, Constanța, Pitești, Brașov and Râmnicu Vâcea. The study, 
even though it was preliminary, was conducted between October 3, 2019 and November 10, 2019. The gender distribution of the subjects involved in the study was as follows: 20 of the subjects $(57.14 \%)$ were female, while the remaining 15 subjects $(42.86 \%)$ were male (Fig. 1).

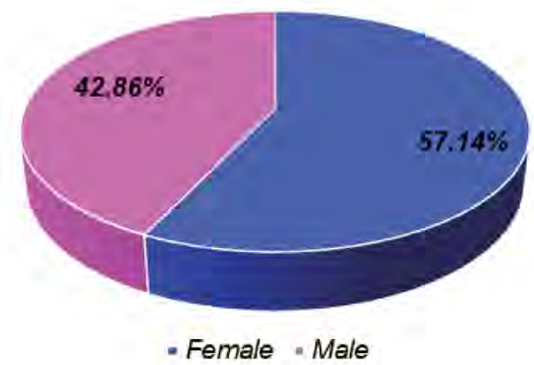

FIGURE 1. Gender distribution of patients involved in the study

The questionnaire used to carry out the study was the following:

1. What are the essential stages in the growth flow of each individual, which involves the temporary dentition? a. The stage named and the age immediately after birth; $\boldsymbol{b}$. The stage called the age of the temporary dentition; $c$. The stage of mixed dentition; d. Age period after 12 years? Correct answers: $b, c$.

2. How is the oral and dental hygiene, until the age of 6 years? a. It is very easy to perform, due to the distributive attention of the child; $\boldsymbol{b}$. It is very difficult to achieve, because of the scattered attention of the child; $c$. It is easy for the child to set the brushing reflex at a certain time of day. Correct answers: $b, c$.

3. How is the presence of dental caries observed during the preschool age? a. With an important dysfunctional role in the imbalance of the dento-maxillary system; b. Without importance in establishing the balance of the dento-maxillary system; $c$. With relative importance in maintaining the balance of the dento-maxillary system. Correct answer: $a$.

4. The period of the mixed dentition (age 6-12 years) represents the stage of changing the temporary dentition with the permanent dentition and comprises 2 phases? $\boldsymbol{a}$. In the period of 6-9 years, the stage of changes in the anterior area takes place; b. Between 6-9 years, the phase of changes in the lateral areas takes place; c. Between 9-12 years, the stage of changes in the lateral zones takes place, influenced by the eruption of the molar of 12 years; $d$. Between 9-12 years, is the stage of changes in the anterior area, influenced by the eruption of the molar of 12 years. Correct answers: $\boldsymbol{a}, \boldsymbol{c}$.
5. In the mixed dentition stage, the complicated dental lesions existing both at the temporary dentition and at the permanent dentition, must meet the following conditions? a. It forces to a correct morphofunctional restoration; b. It must have resistance over time, being important in the development of the dental-maxillary system; $c$. Dental extraction must be performed and the resulting edentation must be rehabilitated with implant-supported prosthetic restorations? Correct answers: $\boldsymbol{a}, \boldsymbol{b}$.

6. In order to prevent the mesial migrations of the distal teeth, it is recommended? a. Treatment and preservation of the temporary dentition as far as possible, (including retained roots, when the situation permits); $\boldsymbol{b}$. Performing temporary tooth extractions, at the smallest dental and/or periodontal disease; c. Applying of some immobilizing teeth devices? Correct answer: $a$.

\section{RESULTS AND DISCUSSIONS}

Regarding the essential stages in the growth flow that involves the temporary dentition most of the respondents (28 representing $80 \%$ ) answered correctly - variants $\mathrm{b}$ and $\mathrm{c}$ (the temporary dentition and the mixed dentition stage). Only 7 respondents (representing 20\%) considered as correct version also the period after 12 years (Fig. 2).

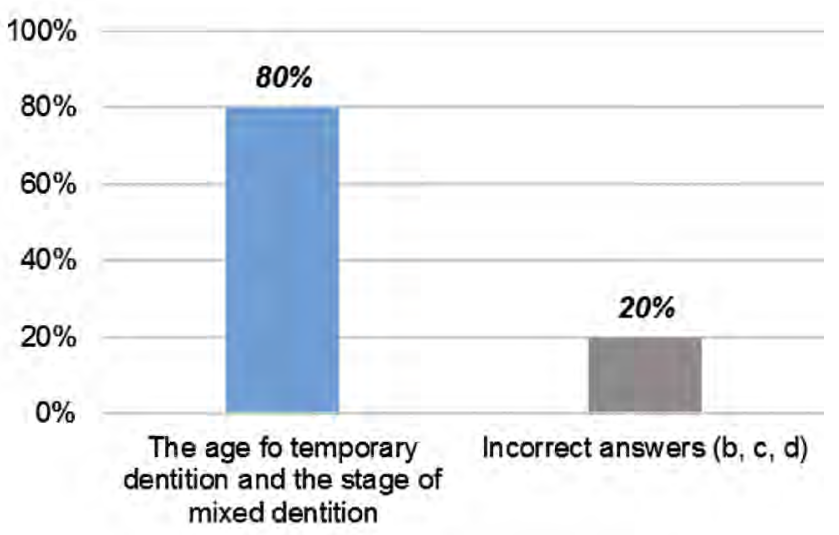

FIGURE 2. Knowledge of the essential stages in the growth flow that involves the temporary dentition

Concerning the oral and dental hygiene up to the age of 6 all practitioners answered correctly - variants $b$ and $c$ (the fact that it is difficult to achieve but can be established as a reflex gesture at a certain time of the day).

For the question related to the presence of dental caries during the preschool age, most of the doctors included in the study ( 25 representing $71.42 \%$ ) answered correctly - an important role in the imbalance of the dento-maxillary system, while 5 doctors 


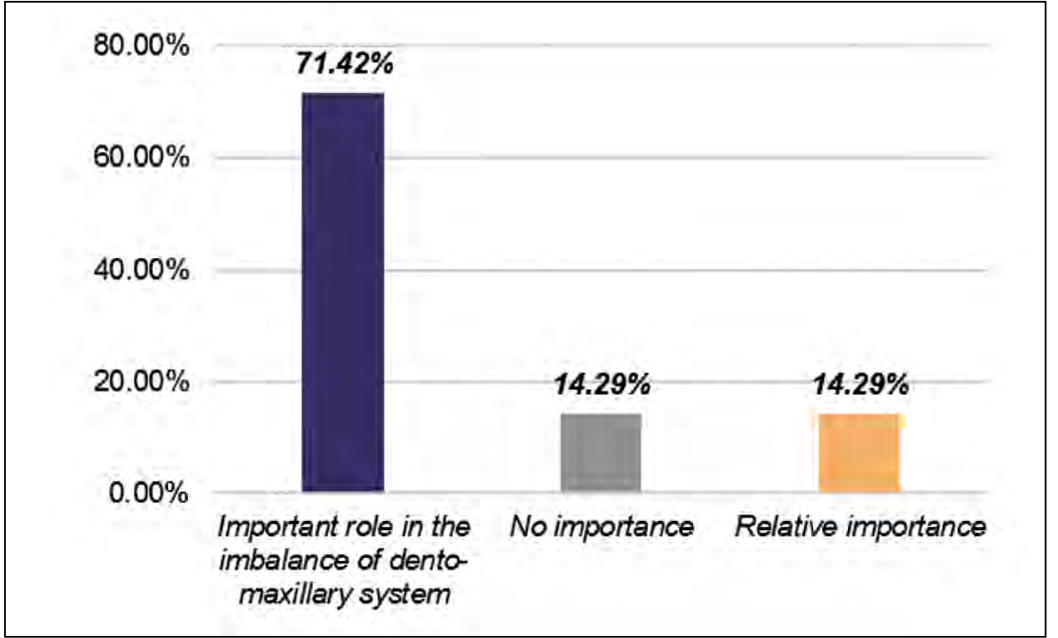

FIGURE 3. The importance of caries during the preschool age (representing 14.29\%) considers it to be of no importance and 5 other doctors consider it to be of relative importance (Fig. 3).

About the two phases of changing the temporary dentition with permanent dentition most of the respondents (30 representing 85.71\%) correctly answered respectively 6-9 years changes in the anterior area and 9-12 years changes in the lateral area. The remaining 5 respondents (representing 14.29\%) incorrectly answered variants c and d (Fig. 4).
For the fifth question related to complicated dental injuries in the mixed dentition all the specialists involved in the study answered correctly variants $a$ and $b$.

At the last point of the questionnaire regarding the measures to be taken to prevent mesial migrations of the distal teeth, the vast majority of respondents (32 representing 91.43\%) answered correctly - variant a, while the remaining 3 respondents (representing 8.57 \%) answered incorrectly - variant b (Fig. 5).
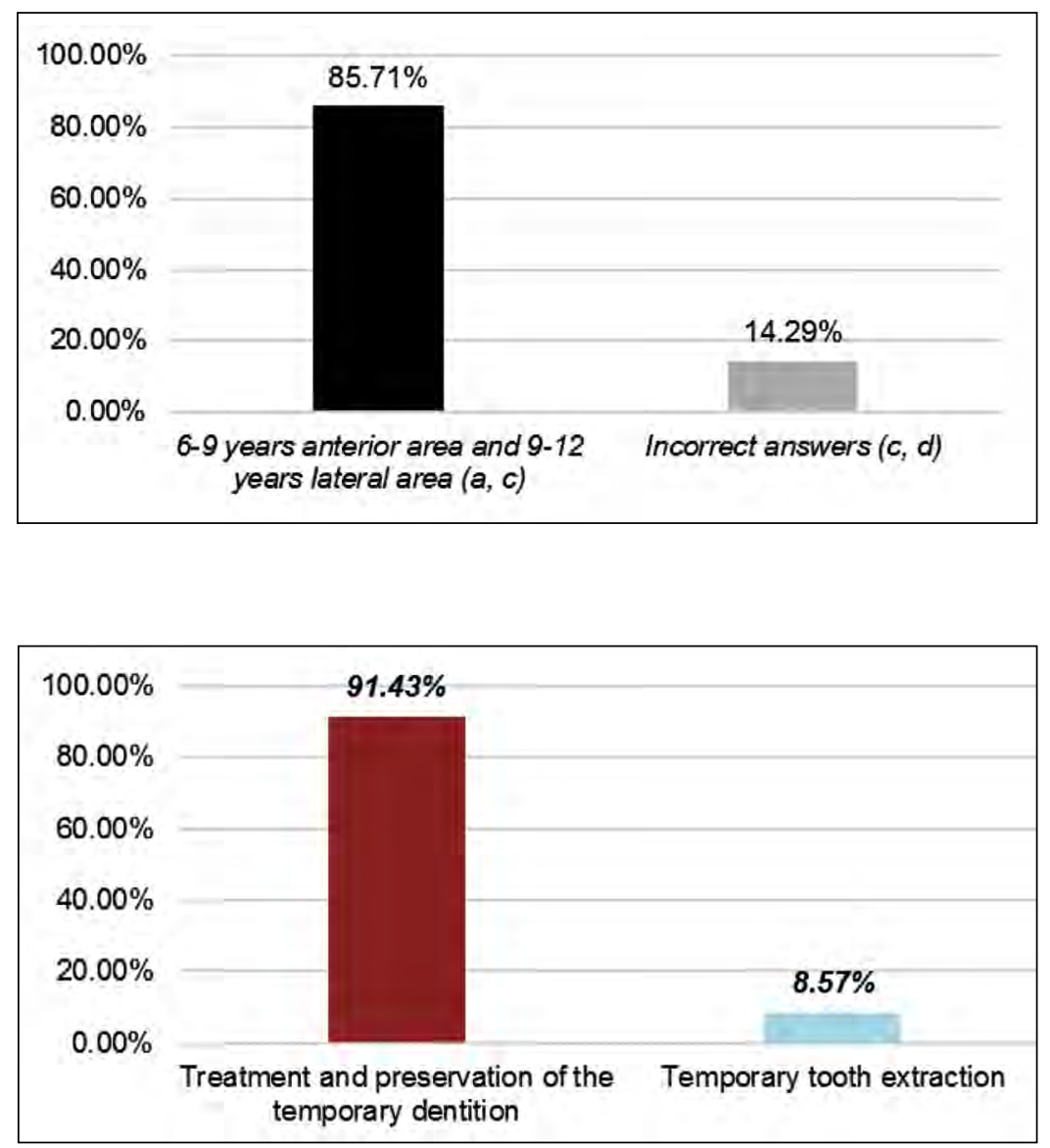

FIGURE 4. Knowledge of the changing phases of the temporary dentition with and permanent dentition
FIGURE 5. Prevention of mesial migrations of distal teeth 


\section{CONCLUSIONS}

Based on the study of the results we can conclude some aspects.

In general, due to the lack of attention or a scattered attention of the child up to 6 years (preschool age), it is difficult to teach him the correct brushing technique. Instead, it is relatively simple to set the brushing reflex at a certain time of day. As the child's attention may be focused, he or she may be explained by a close person the purpose and manner of the brushing.

In the curative therapy of the dental lesions in the temporary dentition, especially before the eruption of the molars of 6 years (the first permanent teeth erupted on the arch), it is important to consider the important role in the development of the dento-maxillary system, of teeth shape reproduction, but also of the rendering the actual relationship of the dental arches.

The execution of therapeutic acts in the treatment of dental lesions of temporary teeth, must be done with the same attention and rigor, as in the case of permanent dentition.

\section{REFERENCES}

1. Gall II. Asistența stomatologică. Ed. Didactică și Pedagogică, București, 1971.

2. Miyasaki-Ching CM. Elemente clinice de stomatologie. Ed. All Educational, București, 2001.

3. Zarnea L. Pedodonție. Ed. Didactică și Pedagogică, București, 1993.

4. Rosenstiel SF, Land MF, Fujimoto J. Contemporary fixed prosthodontics. 4th Edition. Mosby Elsevier, St. Louis, 2006.
The correct preservation of the temporary teeth in the support area, are the basis of the active dental follow-up care in both the state offices from middle school, high school or university educational units, as well as in the private dental offices, the follow-up care that aims to ensure the development of the dental-maxillary system in evolution.

The dentist (performing pediatric dentistry), in the case of complicated dental injuries existing both at the level of the temporary dentition and at the level of the permanent dentition, will consider the general state of the patient and will not keep a septic outbreak where there is the risk of a disease at a distance. In this case, the dentist will choose the dental extraction when indicated, but will ensure the evolution of the normal relationships of the arches, by maintaining space.

\section{Acknowledgement}

In this article, all the authors have equal contribution with the first author.

Conflict of interest: none declared Financial support: none declared

5. Anusavice KJ. Dental materials. Philips' Science 11th Edition. Saunders Elsevier, St. Louis, 2003.

6. Luca R. Pedodonție (Vol. I). București: Ed. Cermaprint, 2003.

7. Luca R. Pedodontie (Vol. II). Bucuresti: Ed. Cermaprint, 2003.

8. Luca R. Pedodonție (Vol. III). București: Ed. Cermaprint, 2013. 